BABAR-CONF-04/007

SLAC-PUB-10626

July 2004

\title{
Measurement of the Inclusive Electron Spectrum in Charmless Semileptonic $B$ Decays Near the Kinematic Endpoint and Determination of $\left|V_{u b}\right|$
}

\author{
The BABAR Collaboration
}

August 16, 2004

\begin{abstract}
We study the inclusive electron spectrum of $B \rightarrow X_{u} e \nu$ decays near the kinematic limit for $B \rightarrow$ $X_{c} e \nu$ transitions using a sample of 88 million $\Upsilon(4 S)$ decays recorded by the BABAR detector at the PEP II $e^{+} e^{-}$- storage rings. For the electron momentum interval of $2.0-2.6 \mathrm{GeV} / c$ the partial branching fraction is measured to be $\Delta \mathcal{B}\left(B \rightarrow X_{u} e \nu\right)=\left(0.480 \pm 0.029_{\text {stat }} \pm 0.053_{\text {syst }}\right) \times 10^{-3}$. Combining this result on $\Delta \mathcal{B}$ with measurements of the inclusive photon spectrum in $B \rightarrow X_{s} \gamma$ transition we find $\left|V_{u b}\right|=\left(3.94 \pm 0.25_{\text {exp }} \pm 0.37_{f_{u}} \pm 0.19_{\text {theory }}\right) \times 10^{-3}$, where the first error represents the combined statistical and systematic experimental uncertainty of the partial branching fraction measurement, the second refers to the uncertainty of the determination of the fraction $f_{u}$ of the inclusive electron spectrum that falls within our cuts, and the third error is due to theoretical uncertainties in the QCD corrections, plus the uncertainty in the b-quark mass. All results are preliminary.
\end{abstract}

Submitted to the $32^{\text {nd }}$ International Conference on High-Energy Physics, ICHEP 04, 16 August - 22 August 2004, Beijing, China

Stanford Linear Accelerator Center, Stanford University, Stanford, CA 94309

Work supported in part by Department of Energy contract DE-AC03-76SF00515. 
The BABAR Collaboration,

B. Aubert, R. Barate, D. Boutigny, F. Couderc, J.-M. Gaillard, A. Hicheur, Y. Karyotakis, J. P. Lees, V. Tisserand, A. Zghiche

Laboratoire de Physique des Particules, F-74941 Annecy-le-Vieux, France

A. Palano, A. Pompili

Università di Bari, Dipartimento di Fisica and INFN, I-70126 Bari, Italy

J. C. Chen, N. D. Qi, G. Rong, P. Wang, Y. S. Zhu

Institute of High Energy Physics, Beijing 100039, China

G. Eigen, I. Ofte, B. Stugu

University of Bergen, Inst. of Physics, N-5007 Bergen, Norway

G. S. Abrams, A. W. Borgland, A. B. Breon, D. N. Brown, J. Button-Shafer, R. N. Cahn, E. Charles, C. T. Day, M. S. Gill, A. V. Gritsan, Y. Groysman, R. G. Jacobsen, R. W. Kadel, J. Kadyk, L. T. Kerth, Yu. G. Kolomensky, G. Kukartsev, G. Lynch, L. M. Mir, P. J. Oddone, T. J. Orimoto, M. Pripstein, N. A. Roe, M. T. Ronan, V. G. Shelkov, W. A. Wenzel

Lawrence Berkeley National Laboratory and University of California, Berkeley, CA 94720, USA

M. Barrett, K. E. Ford, T. J. Harrison, A. J. Hart, C. M. Hawkes, S. E. Morgan, A. T. Watson University of Birmingham, Birmingham, B15 2TT, United Kingdom

M. Fritsch, K. Goetzen, T. Held, H. Koch, B. Lewandowski, M. Pelizaeus, M. Steinke Ruhr Universität Bochum, Institut für Experimentalphysik 1, D-44780 Bochum, Germany

J. T. Boyd, N. Chevalier, W. N. Cottingham, M. P. Kelly, T. E. Latham, F. F. Wilson University of Bristol, Bristol BS8 1TL, United Kingdom

T. Cuhadar-Donszelmann, C. Hearty, N. S. Knecht, T. S. Mattison, J. A. McKenna, D. Thiessen University of British Columbia, Vancouver, BC, Canada V6T $1 Z 1$

A. Khan, P. Kyberd, L. Teodorescu Brunel University, Uxbridge, Middlesex UB8 3PH, United Kingdom

A. E. Blinov, V. E. Blinov, V. P. Druzhinin, V. B. Golubev, V. N. Ivanchenko, E. A. Kravchenko, A. P. Onuchin, S. I. Serednyakov, Yu. I. Skovpen, E. P. Solodov, A. N. Yushkov

Budker Institute of Nuclear Physics, Novosibirsk 630090, Russia

D. Best, M. Bruinsma, M. Chao, I. Eschrich, D. Kirkby, A. J. Lankford, M. Mandelkern, R. K. Mommsen, W. Roethel, D. P. Stoker University of California at Irvine, Irvine, CA 92697, USA

C. Buchanan, B. L. Hartfiel

University of California at Los Angeles, Los Angeles, CA 90024, USA

S. D. Foulkes, J. W. Gary, B. C. Shen, K. Wang

University of California at Riverside, Riverside, CA 92521, USA 
D. del Re, H. K. Hadavand, E. J. Hill, D. B. MacFarlane, H. P. Paar, Sh. Rahatlou, V. Sharma University of California at San Diego, La Jolla, CA 92093, USA

J. W. Berryhill, C. Campagnari, B. Dahmes, O. Long, A. Lu, M. A. Mazur, J. D. Richman, W. Verkerke University of California at Santa Barbara, Santa Barbara, CA 93106, USA

T. W. Beck, A. M. Eisner, C. A. Heusch, J. Kroseberg, W. S. Lockman, G. Nesom, T. Schalk, B. A. Schumm, A. Seiden, P. Spradlin, D. C. Williams, M. G. Wilson

University of California at Santa Cruz, Institute for Particle Physics, Santa Cruz, CA 95064, USA

J. Albert, E. Chen, G. P. Dubois-Felsmann, A. Dvoretskii, D. G. Hitlin, I. Narsky, T. Piatenko, F. C. Porter, A. Ryd, A. Samuel, S. Yang

California Institute of Technology, Pasadena, CA 91125, USA

S. Jayatilleke, G. Mancinelli, B. T. Meadows, M. D. Sokoloff

University of Cincinnati, Cincinnati, OH 45221, USA

T. Abe, F. Blanc, P. Bloom, S. Chen, W. T. Ford, U. Nauenberg, A. Olivas, P. Rankin, J. G. Smith, J. Zhang, L. Zhang University of Colorado, Boulder, CO 80309, USA

A. Chen, J. L. Harton, A. Soffer, W. H. Toki, R. J. Wilson, Q. Zeng Colorado State University, Fort Collins, CO 80523, USA

D. Altenburg, T. Brandt, J. Brose, M. Dickopp, E. Feltresi, A. Hauke, H. M. Lacker, R. Müller-Pfefferkorn, R. Nogowski, S. Otto, A. Petzold, J. Schubert, K. R. Schubert, R. Schwierz, B. Spaan, J. E. Sundermann

Technische Universität Dresden, Institut für Kern- und Teilchenphysik, D-01062 Dresden, Germany

D. Bernard, G. R. Bonneaud, F. Brochard, P. Grenier, S. Schrenk, Ch. Thiebaux, G. Vasileiadis, M. Verderi Ecole Polytechnique, LLR, F-91128 Palaiseau, France

D. J. Bard, P. J. Clark, D. Lavin, F. Muheim, S. Playfer, Y. Xie

University of Edinburgh, Edinburgh EH9 3JZ, United Kingdom

M. Andreotti, V. Azzolini, D. Bettoni, C. Bozzi, R. Calabrese, G. Cibinetto, E. Luppi, M. Negrini, L. Piemontese, A. Sarti

Università di Ferrara, Dipartimento di Fisica and INFN, I-44100 Ferrara, Italy

E. Treadwell

Florida A $6 M$ University, Tallahassee, FL 3230\%, USA

F. Anulli, R. Baldini-Ferroli, A. Calcaterra, R. de Sangro, G. Finocchiaro, P. Patteri, I. M. Peruzzi, M. Piccolo, A. Zallo

Laboratori Nazionali di Frascati dell'INFN, I-00044 Frascati, Italy

A. Buzzo, R. Capra, R. Contri, G. Crosetti, M. Lo Vetere, M. Macri, M. R. Monge, S. Passaggio, C. Patrignani, E. Robutti, A. Santroni, S. Tosi

Università di Genova, Dipartimento di Fisica and INFN, I-16146 Genova, Italy

S. Bailey, G. Brandenburg, K. S. Chaisanguanthum, M. Morii, E. Won

Harvard University, Cambridge, MA 02138, USA 
R. S. Dubitzky, U. Langenegger

Universität Heidelberg, Physikalisches Institut, Philosophenweg 12, D-69120 Heidelberg, Germany

W. Bhimji, D. A. Bowerman, P. D. Dauncey, U. Egede, J. R. Gaillard, G. W. Morton, J. A. Nash, M. B. Nikolich, G. P. Taylor Imperial College London, London, SWr 2AZ, United Kingdom

M. J. Charles, G. J. Grenier, U. Mallik

University of Iowa, Iowa City, IA 52242, USA

J. Cochran, H. B. Crawley, J. Lamsa, W. T. Meyer, S. Prell, E. I. Rosenberg, A. E. Rubin, J. Yi Iowa State University, Ames, IA 50011-3160, USA

M. Biasini, R. Covarelli, M. Pioppi

Università di Perugia, Dipartimento di Fisica and INFN, I-06100 Perugia, Italy

M. Davier, X. Giroux, G. Grosdidier, A. Höcker, S. Laplace, F. Le Diberder, V. Lepeltier, A. M. Lutz, T. C. Petersen, S. Plaszczynski, M. H. Schune, L. Tantot, G. Wormser

Laboratoire de l'Accélérateur Linéaire, F-91898 Orsay, France

C. H. Cheng, D. J. Lange, M. C. Simani, D. M. Wright

Lawrence Livermore National Laboratory, Livermore, CA 94550, USA

A. J. Bevan, C. A. Chavez, J. P. Coleman, I. J. Forster, J. R. Fry, E. Gabathuler, R. Gamet, D. E. Hutchcroft, R. J. Parry, D. J. Payne, R. J. Sloane, C. Touramanis

University of Liverpool, Liverpool L69 72E, United Kingdom

J. J. Back, ${ }^{1}$ C. M. Cormack, P. F. Harrison, ${ }^{1}$ F. Di Lodovico, G. B. Mohanty ${ }^{1}$

Queen Mary, University of London, E1 4NS, United Kingdom

C. L. Brown, G. Cowan, R. L. Flack, H. U. Flaecher, M. G. Green, P. S. Jackson, T. R. McMahon, S. Ricciardi, F. Salvatore, M. A. Winter

University of London, Royal Holloway and Bedford New College, Egham, Surrey TW20 0EX, United Kingdom

D. Brown, C. L. Davis

University of Louisville, Louisville, KY 40292, USA

J. Allison, N. R. Barlow, R. J. Barlow, P. A. Hart, M. C. Hodgkinson, G. D. Lafferty, A. J. Lyon, J. C. Williams

University of Manchester, Manchester M13 9PL, United Kingdom

A. Farbin, W. D. Hulsbergen, A. Jawahery, D. Kovalskyi, C. K. Lae, V. Lillard, D. A. Roberts

University of Maryland, College Park, MD 20742, USA

G. Blaylock, C. Dallapiccola, K. T. Flood, S. S. Hertzbach, R. Kofler, V. B. Koptchev, T. B. Moore, S. Saremi, H. Staengle, S. Willocq

University of Massachusetts, Amherst, MA 01003, USA

${ }^{1}$ Now at Department of Physics, University of Warwick, Coventry, United Kingdom 
R. Cowan, G. Sciolla, S. J. Sekula, F. Taylor, R. K. Yamamoto

Massachusetts Institute of Technology, Laboratory for Nuclear Science, Cambridge, MA 02139, USA

D. J. J. Mangeol, P. M. Patel, S. H. Robertson

McGill University, Montréal, QC, Canada H3A 2T8

A. Lazzaro, V. Lombardo, F. Palombo

Università di Milano, Dipartimento di Fisica and INFN, I-20133 Milano, Italy

J. M. Bauer, L. Cremaldi, V. Eschenburg, R. Godang, R. Kroeger, J. Reidy, D. A. Sanders, D. J. Summers, H. W. Zhao

University of Mississippi, University, MS 3867\%, USA

S. Brunet, D. Côté, P. Taras

Université de Montréal, Laboratoire René J. A. Lévesque, Montréal, QC, Canada H3C 3J7

H. Nicholson

Mount Holyoke College, South Hadley, MA 01075, USA

N. Cavallo, ${ }^{2}$ F. Fabozzi, ${ }^{2}$ C. Gatto, L. Lista, D. Monorchio, P. Paolucci, D. Piccolo, C. Sciacca

Università di Napoli Federico II, Dipartimento di Scienze Fisiche and INFN, I-80126, Napoli, Italy

M. Baak, H. Bulten, G. Raven, H. L. Snoek, L. Wilden

NIKHEF, National Institute for Nuclear Physics and High Energy Physics, NL-1009 DB Amsterdam, The Netherlands

C. P. Jessop, J. M. LoSecco

University of Notre Dame, Notre Dame, IN 46556, USA

T. Allmendinger, K. K. Gan, K. Honscheid, D. Hufnagel, H. Kagan, R. Kass, T. Pulliam, A. M. Rahimi, R. Ter-Antonyan, Q. K. Wong

Ohio State University, Columbus, OH 43210, USA

J. Brau, R. Frey, O. Igonkina, C. T. Potter, N. B. Sinev, D. Strom, E. Torrence

University of Oregon, Eugene, OR 97403, USA

F. Colecchia, A. Dorigo, F. Galeazzi, M. Margoni, M. Morandin, M. Posocco, M. Rotondo, F. Simonetto, R. Stroili, G. Tiozzo, C. Voci

Università di Padova, Dipartimento di Fisica and INFN, I-35131 Padova, Italy

M. Benayoun, H. Briand, J. Chauveau, P. David, Ch. de la Vaissière, L. Del Buono, O. Hamon, M. J. J. John, Ph. Leruste, J. Malcles, J. Ocariz, M. Pivk, L. Roos, S. T'Jampens, G. Therin

Universités Paris VI et VII, Laboratoire de Physique Nucléaire et de Hautes Energies, F-75252 Paris, France

P. F. Manfredi, V. Re

Università di Pavia, Dipartimento di Elettronica and INFN, I-27100 Pavia, Italy

\footnotetext{
${ }^{2}$ Also with Università della Basilicata, Potenza, Italy
} 


\author{
P. K. Behera, L. Gladney, Q. H. Guo, J. Panetta \\ University of Pennsylvania, Philadelphia, PA 19104, USA
}

C. Angelini, G. Batignani, S. Bettarini, M. Bondioli, F. Bucci, G. Calderini, M. Carpinelli, F. Forti, M. A. Giorgi, A. Lusiani, G. Marchiori, F. Martinez-Vidal, ${ }^{3}$ M. Morganti, N. Neri, E. Paoloni, M. Rama, G. Rizzo, F. Sandrelli, J. Walsh

Università di Pisa, Dipartimento di Fisica, Scuola Normale Superiore and INFN, I-56127 Pisa, Italy

M. Haire, D. Judd, K. Paick, D. E. Wagoner

Prairie View A\&M University, Prairie View, TX 77446, USA

N. Danielson, P. Elmer, Y. P. Lau, C. Lu, V. Miftakov, J. Olsen, A. J. S. Smith, A. V. Telnov

Princeton University, Princeton, NJ 08544, USA

F. Bellini, G. Cavoto, ${ }^{4}$ R. Faccini, F. Ferrarotto, F. Ferroni, M. Gaspero, L. Li Gioi, M. A. Mazzoni, S. Morganti, M. Pierini, G. Piredda, F. Safai Tehrani, C. Voena Università di Roma La Sapienza, Dipartimento di Fisica and INFN, I-00185 Roma, Italy

S. Christ, G. Wagner, R. Waldi

Universität Rostock, D-18051 Rostock, Germany

T. Adye, N. De Groot, B. Franek, N. I. Geddes, G. P. Gopal, E. O. Olaiya

Rutherford Appleton Laboratory, Chilton, Didcot, Oxon, OX11 0QX, United Kingdom

R. Aleksan, S. Emery, A. Gaidot, S. F. Ganzhur, P.-F. Giraud, G. Hamel de Monchenault, W. Kozanecki, M. Legendre, G. W. London, B. Mayer, G. Schott, G. Vasseur, Ch. Yèche, M. Zito

DSM/Dapnia, CEA/Saclay, F-91191 Gif-sur-Yvette, France

M. V. Purohit, A. W. Weidemann, J. R. Wilson, F. X. Yumiceva

University of South Carolina, Columbia, SC 29208, USA

D. Aston, R. Bartoldus, N. Berger, A. M. Boyarski, O. L. Buchmueller, R. Claus, M. R. Convery, M. Cristinziani, G. De Nardo, D. Dong, J. Dorfan, D. Dujmic, W. Dunwoodie, E. E. Elsen, S. Fan,

R. C. Field, T. Glanzman, S. J. Gowdy, T. Hadig, V. Halyo, C. Hast, T. Hryn'ova, W. R. Innes,

M. H. Kelsey, P. Kim, M. L. Kocian, D. W. G. S. Leith, J. Libby, S. Luitz, V. Luth, H. L. Lynch,

H. Marsiske, R. Messner, D. R. Muller, C. P. O'Grady, V. E. Ozcan, A. Perazzo, M. Perl, S. Petrak,

B. N. Ratcliff, A. Roodman, A. A. Salnikov, R. H. Schindler, J. Schwiening, G. Simi, A. Snyder, A. Soha, J. Stelzer, D. Su, M. K. Sullivan, J. Va'vra, S. R. Wagner, M. Weaver, A. J. R. Weinstein,

W. J. Wisniewski, M. Wittgen, D. H. Wright, A. K. Yarritu, C. C. Young

Stanford Linear Accelerator Center, Stanford, CA 94309, USA

P. R. Burchat, A. J. Edwards, T. I. Meyer, B. A. Petersen, C. Roat

Stanford University, Stanford, CA 94305-4060, USA

S. Ahmed, M. S. Alam, J. A. Ernst, M. A. Saeed, M. Saleem, F. R. Wappler

State University of New York, Albany, NY 12222, USA

\footnotetext{
${ }^{3}$ Also with IFIC, Instituto de Física Corpuscular, CSIC-Universidad de Valencia, Valencia, Spain

${ }^{4}$ Also with Princeton University, Princeton, USA
} 
W. Bugg, M. Krishnamurthy, S. M. Spanier

University of Tennessee, Knoxville, TN 37996, USA

R. Eckmann, H. Kim, J. L. Ritchie, A. Satpathy, R. F. Schwitters

University of Texas at Austin, Austin, TX 78712, USA

J. M. Izen, I. Kitayama, X. C. Lou, S. Ye

University of Texas at Dallas, Richardson, TX 75083, USA

F. Bianchi, M. Bona, F. Gallo, D. Gamba

Università di Torino, Dipartimento di Fisica Sperimentale and INFN, I-10125 Torino, Italy

L. Bosisio, C. Cartaro, F. Cossutti, G. Della Ricca, S. Dittongo, S. Grancagnolo, L. Lanceri, P. Poropat, ${ }^{5}$ L. Vitale, G. Vuagnin

Università di Trieste, Dipartimento di Fisica and INFN, I-34127 Trieste, Italy

R. S. Panvini

Vanderbilt University, Nashville, TN 37235, USA

Sw. Banerjee, C. M. Brown, D. Fortin, P. D. Jackson, R. Kowalewski, J. M. Roney, R. J. Sobie University of Victoria, Victoria, BC, Canada V8W $3 P 6$

H. R. Band, B. Cheng, S. Dasu, M. Datta, A. M. Eichenbaum, M. Graham, J. J. Hollar, J. R. Johnson, P. E. Kutter, H. Li, R. Liu, A. Mihalyi, A. K. Mohapatra, Y. Pan, R. Prepost, P. Tan, J. H. von Wimmersperg-Toeller, J. Wu, S. L. Wu, Z. Yu

University of Wisconsin, Madison, WI 53706, USA

M. G. Greene, H. Neal

Yale University, New Haven, CT 06511, USA

\footnotetext{
${ }^{5}$ Deceased
} 


\section{Introduction}

The increasingly precise measurements of $\mathrm{CP}$ asymmetries in $B$-meson decays allow for stringent experimental tests of the Standard Model mechanism for CP violation [1] via the non-trivial phase in the Cabibbo-Kobayashi-Maskawa matrix. Improved measurements of CKM element $\left|V_{u b}\right|$, the coupling of the $b$ quark to the $u$ quark, will enhance the sensitivity of such experimental tests.

The extraction of $\left|V_{u b}\right|$ is a challenge, both theoretically and experimentally. Experimentally, the principal challenge is to separate the signal $B \rightarrow X_{u} e \nu$ decays from the 50 times larger $B \rightarrow X_{c} e \nu$ background. This is achieved by selecting regions of phase space in which the background is highly suppressed. In this paper we present a measurement of the inclusive electron spectrum for charmless semileptonic $B$ decays, averaged over charged and neutral $B$ mesons, near the kinematic endpoint. In the rest frame of the $B$ meson, the kinematic endpoint of the electron spectrum for the dominant $B \rightarrow X_{c} e \nu$ decays is $\sim 2.3 \mathrm{GeV} / c$ and $\sim 2.6 \mathrm{GeV} / c$ for $B \rightarrow X_{u} e \nu$ decays. The finite momentum of the $B$ mesons in the $\Upsilon(4 S)$ decays causes additional spread of the electron momenta of $\sim 200 \mathrm{MeV} / c$, extending the endpoints to higher momenta. A narrow interval of about $300 \mathrm{MeV} / c$ remains dominated by electrons from $B \rightarrow X_{u} e \nu$ transitions, and this allows for a relatively precise measurement of the partial branching fractions in an interval that covers approximately $10-15 \%$ of the total electron spectrum for charmless semileptonic $B$ decays free from significant $B \bar{B}$ background. In this analysis we extend the interval for signal extraction up to $600 \mathrm{MeV} / c$ covering $\sim 30 \%$ of the total electron spectrum.

Theoretically the weak decay rate for $b \rightarrow u \ell \nu$ can be easily calculated at the parton level. It is proportional to $\left|V_{u b}\right|^{2}$ and $m_{b}^{5}$, where $m_{b}$ refers to the $b$ quark mass. To relate the $B$ meson decay rate to $\left|V_{u b}\right|$, the parton-level calculations have to be corrected for perturbative and nonperturbative QCD effects. These corrections can be calculated using operator-product expansions in powers of $1 / m_{b}$ and $\alpha_{s}[2]$. However, near the kinematic endpoint in the lepton spectrum, these calculations break down because the spectra are affected by the "shape function", i.e., the distribution of the $b$-quark momentum inside the $B$ meson [3], in addition to weak annihilation and other effects. Thus extrapolation from the limited momentum range near the endpoint to the full

spectrum is a difficult task. At present, the shape function cannot be calculated, but it should be a universal property of the $B$ meson. To leading order it must be the same for all $b \rightarrow q$ transitions (here $q$ represents any light quark). It has been proposed [4], [5] that $\left|V_{u b}\right|$ can be extracted by comparing the high-energy end of the lepton spectrum with the high end of the photon spectrum in $b \rightarrow s \gamma$ transitions, thus reducing the theoretical uncertainty on the shape function. Inclusive measurements of the photon spectrum are very challenging, but two results are now available [6], [7]. This analysis is based on the same method as previous measurements of the lepton spectrum near the endpoint [8] (ARGUS), [9], [10](CLEO). The measurement of the partial branching fraction for charmless semileptonic decays and the extraction of $\left|V_{u b}\right|$ presented here are updates of preliminary results [11] based on one-fourth the data.

\section{Data Sample, Detector, and Simulation}

The data used in this analysis were recorded with the BABAR detector at the PEP-II energyasymmetric $e^{+} e^{-}$collider. The data sample of 88.4 million $B \bar{B}$ events, corresponding to an integrated luminosity of $80.4 \mathrm{fb}^{-1}$, was collected at the $\Upsilon(4 S)$ resonance; an additional sample of $9.5 \mathrm{fb}^{-1}$ was recorded at a center-of-mass energy just below the $B \bar{B}$ threshold. The second data set is used for subtraction of the non- $B \bar{B}$ contributions from the data collected on the $\Upsilon(4 S)$ 
resonance. The relative normalization of the two data samples has been derived from luminosity measurements, which are based on the number of detected $\mu^{+} \mu^{-}$pairs and the QED cross section for $e^{+} e^{-} \rightarrow \mu^{+} \mu^{-}$production, adjusted for the small difference in center-of-mass energy.

The BABAR detector has been described in detail elsewhere [12]. The most important components for this study are the charged-particle tracking system, consisting of a five-layer silicon detector and a 40-layer drift chamber, and the electromagnetic calorimeter assembled from 6580 $\mathrm{CsI}(\mathrm{Tl})$ crystals. These detector components are embedded in a $1.5 \mathrm{~T}$ solenoidal magnetic field. Electron candidates are selected on the basis of the ratio of the energy detected in the calorimeter to the track momentum, the calorimeter shower shape, the energy loss in the drift chamber, and the angle reconstructed in the ring-imaging Cherenkov detector.

The electron identification efficiency and the probabilities to misidentify a pion, kaon, or proton as an electron have been measured as a function of laboratory momentum and angles [13] with clean samples of tracks that were selected from data. This experimental information is used in the Monte Carlo simulation to improve the agreement with the data. Within the acceptance of the calorimeter, defined by the polar angle in the laboratory frame, $-0.72<\cos \theta_{l a b}<0.92$, the average electron efficiency is $92 \%$. The average hadron misidentification rate is about $0.1 \%$.

Tracking efficiencies and resolution have been studied in great detail. Comparisons with the simulation have revealed small differences, which have been taken into account. No significant impact of non-Gaussian resolution tails has been found for high momentum tracks contributing to the endpoint region.

We use Monte Carlo simulation of $B \bar{B}$ events to estimate signal efficiencies and background distributions. Most of the branching fractions for hadronic $B$ and $D$ decays and form factors are based on values reported in the Review of Particle Physics [14]. Charmless semileptonic decays, $B \rightarrow X_{u} \ell \nu$, are simulated as a mixture of three-body decays $\left(X_{u}=\pi, \eta, \rho, \omega, \ldots\right)$ based on the ISGW2 model [15], and decays to hadronic states $X_{u}$, with masses $m_{X_{u}}$ extending from $2 m_{\pi}$ to about $3.5 \mathrm{GeV} / c^{2}$, according to the prescription of Ref. [16]. The hadronization of $X_{u}$ is performed by JETSET [17]. The motion of the $b$ quark inside the $B$ meson is implemented with the shape function parameterization given in [16]. The low-mass resonant decays are mixed with the nonresonant states in such a way as to retain the cumulative distribution in the hadron mass $m_{X_{u}}$ as predicted by the non-resonant model of [16].

For the simulation of the dominant $B \rightarrow X_{c} \ell \nu$ decays we have chosen a variety of models. We use ISGW2 [15] for $B \rightarrow D \ell \nu$ and the various decays to higher mass $D^{* *}$ resonances, adopt a prescription by Goity-Roberts [18] for the non-resonant $B \rightarrow D^{(*)} \pi \ell \nu$ decays, and use an HQET parameterization [19] of the form factors for $B \rightarrow D^{*} \ell \nu$. For these form factors we use the recent results from $B A B A R$ [20], which have much smaller uncertainties than the earlier measurements by CLEO [21]. The branching fractions for individual $B \rightarrow X_{c} \ell \nu$ decays are adjusted to match the data (see below).

The Monte Carlo simulations include radiative effects such as bremsstrahlung in the detector material and QED initial and final state radiation. Adjustments for small variation of the beam energy over time have also been included. 


\section{Analysis}

\subsection{Event Selection}

We select semileptonic $B$-decay events by requiring that there be an electron with momentum $p_{e}>1.1 \mathrm{GeV} / c$ in the $\Upsilon(4 S)$ rest frame. Throughout this paper, all quantities are measured in the $\Upsilon(4 S)$ rest frame unless it is specified otherwise. To reject electrons from the decay $J / \psi \rightarrow e^{+} e^{-}$we combine the electron candidate with any second electron of opposite charge and reject the candidate if the invariant mass of the pair falls in the interval $3.00<m_{e e}<3.15 \mathrm{GeV} / c^{2}$.

To suppress background from non- $B \bar{B}$ events, primarily low multiplicity QED (including $\tau^{+} \tau^{-}$ pairs) and $e^{+} e^{-} \rightarrow q \bar{q}$ processes, we veto events with less than four charged tracks. We also require that the ratio of the second to the zeroth Fox-Wolfram moments, $R_{2}=H_{2} / H_{0}$ [22], not exceed 0.5. $R_{2}$ is calculated including all detected charged particles and photons. For events with an electron in the momentum interval of 2.0 to $2.6 \mathrm{GeV} / c$, these two criteria reduce the non- $B \bar{B}$ background by a factor of about 6 , while they retain more than $80 \%$ of the signal events.

In semileptonic $B$ decays, the neutrino carries sizable energy. In events in which the only undetected particle is this neutrino, its energy and direction can be inferred from the missing momentum in the event, $p_{\text {miss }}$. We estimate $p_{\text {miss }}$ from the difference between the four-momentum of the two colliding-beam particles and sum of the four-momenta of all detected particles, charged and neutral. To improve the reconstruction of the missing momentum, we impose a number of requirements on the charged and neutral particles. Charged tracks are required to have a minimum transverse momentum of $0.2 \mathrm{GeV} / c$ and a maximum momentum of $10 \mathrm{GeV} / c$ in the laboratory frame. They are restricted in polar angle to $-0.82<\cos \theta_{l a b}<0.92$ and should originate close to the beam-beam interaction point. The detected energy of an individual photon is required to exceed $30 \mathrm{MeV}$. The selection of semileptonic decays can be greatly enhanced by requiring for the missing momentum that $\left|\vec{p}_{\text {miss }}\right|$ exceed $0.5 \mathrm{GeV} / c$, and that $\vec{p}_{\text {miss }}$ point into the detector fiducial volume, $-0.9<\cos \theta_{\text {miss }}<0.9$, thereby effectively reducing the impact of particle losses close to the beams. Furthermore, since in semileptonic $B$ decays, the neutrino and the electron are emitted preferentially in opposite directions, we require that the angle $\Delta \alpha$ between these two particles fulfill the condition $\cos \Delta \alpha<0.4$. These criteria on the missing momentum reduce the continuum background from QED processes and $e^{+} e^{-} \rightarrow q \bar{q}$ production by an additional factor of 3 , while the signal loss is less than $20 \%$.

The detection efficiencies are estimated by Monte Carlo simulation. With the stated selection criteria the efficiency (including effects of bremsstrahlung) for detecting $B \rightarrow X_{u} e \nu$ decays is close to $50 \%$ and largely independent of the electron momentum between 1.1 and $2.0 \mathrm{GeV} / c$; it gradually decreases above $2.0 \mathrm{GeV} / c$, reaching $35 \%$ at $2.6 \mathrm{GeV} / c$.

\subsection{Background Subtraction}

The spectrum of the highest momentum electron in events selected by the criteria described above is shown in Fig. 1a, separately for data recorded on and below the $\Upsilon(4 S)$ resonance. The data collected on the $\Upsilon(4 S)$ resonance include contributions from $B \bar{B}$ events and non- $B \bar{B}$ background. The latter is measured using off-resonance data, collected below $B \bar{B}$ production threshold, and using on-resonance data above $2.8 \mathrm{GeV} / c$, i.e., above the endpoint for electrons from $B$ decays. The $B \bar{B}$ background to the $B \rightarrow X_{u} e \nu$ spectrum is estimated from Monte Carlo simulation with the normalization of the individual contributions determined by a fit to the total observed spectrum. 


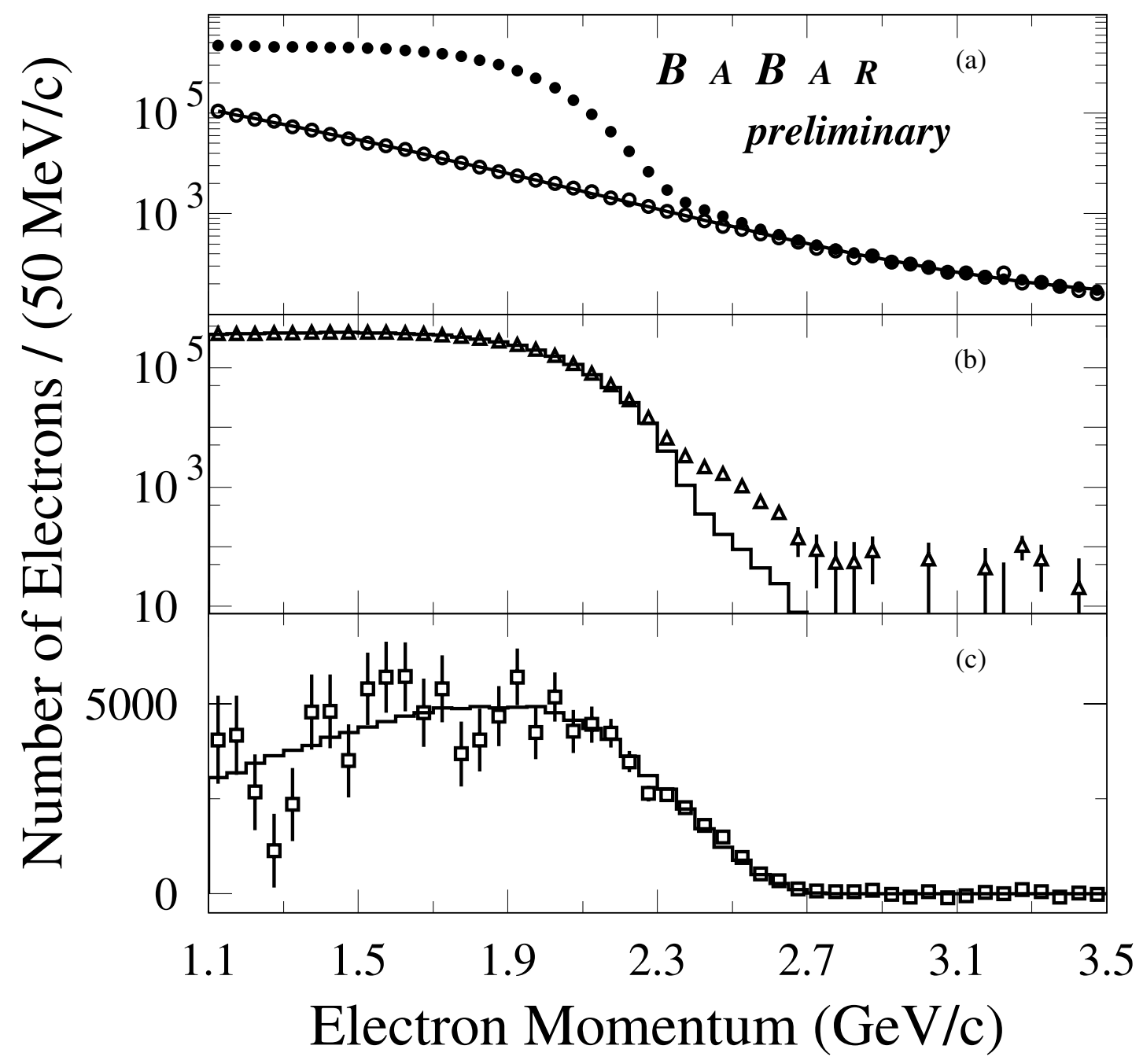

Figure 1: Electron momentum spectrum in the $\Upsilon(4 S)$ rest frame: (a) on-resonance data (solid circles), scaled off-resonance data (open circles). The solid line shows the result of the fit to the non- $B \bar{B}$ events using both on- and off-resonance data in the interval $p_{e}=1.1-3.5 \mathrm{GeV} / c$; (b) on-resonance data after subtraction of the fitted non- $B \bar{B}$ background (open triangles) compared to simulated $B \bar{B}$ background (histogram); (c) on-resonance data after subtraction of all backgrounds (open squares), compared to the simulated $B \rightarrow X_{u} e \nu$ events (histogram). The error bars indicate statistical errors only.

\subsubsection{Non- $B \bar{B} \quad$ Background}

To determine the non- $B \bar{B}$ background we perform a $\chi^{2}$ fit to the off-resonance data, collected below $B \bar{B}$ production threshold, and to on-resonance data in the momentum interval of $2.8 \mathrm{GeV} / c$ to $3.5 \mathrm{GeV} / c$. Since the c.m. energy for the off-resonance data is lower by about $0.4 \%$, we scale 
Table 1: Summary of sample composition: principal backgrounds and remaining signal in units of $10^{3}$ events, as well as the signal efficiencies. The errors are statistical, but for the non- $B \bar{B}$ and $X_{c} e \nu$ backgrounds the uncertainties in the fitted scale factors are included.

\begin{tabular}{lrrrr}
\hline \hline$\Delta p(\mathrm{GeV} / c)$ & $2.0 \div 2.6$ & $2.1 \div 2.6$ & $2.2 \div 2.6$ & \multicolumn{1}{c}{$2.3 \div 2.6$} \\
\hline Total sample & $609.81 \pm 0.78$ & $295.76 \pm 0.54$ & $133.59 \pm 0.37$ & $65.48 \pm 0.26$ \\
\hline Non $-B \bar{B}$ & $142.33 \pm 0.63$ & $105.15 \pm 0.48$ & $74.83 \pm 0.36$ & $50.10 \pm 0.25$ \\
$X_{c} e \nu$ & $423.25 \pm 1.58$ & $160.17 \pm 0.86$ & $39.76 \pm 0.35$ & $4.18 \pm 0.09$ \\
$J / \psi$ and $\psi^{\prime}$ & $5.50 \pm 0.14$ & $3.55 \pm 0.09$ & $2.07 \pm 0.06$ & $1.04 \pm 0.03$ \\
Other $e^{ \pm}$ & $1.57 \pm 0.04$ & $0.61 \pm 0.02$ & $0.23 \pm 0.01$ & $0.07 \pm 0.01$ \\
$\pi$ mis-ID & $1.19 \pm 0.04$ & $0.87 \pm 0.03$ & $0.57 \pm 0.02$ & $0.31 \pm 0.02$ \\
$K$ mis-ID & $0.44 \pm 0.02$ & $0.24 \pm 0.01$ & $0.12 \pm 0.01$ & $0.05 \pm 0.01$ \\
Other mis-ID & $0.24 \pm 0.01$ & $0.13 \pm 0.01$ & $0.07 \pm 0.01$ & $0.03 \pm 0.01$ \\
Other $X_{u} e \nu$ & $1.33 \pm 0.07$ & $0.54 \pm 0.03$ & $0.16 \pm 0.01$ & $0.03 \pm 0.01$ \\
\hline$X_{u} e \nu$ signal & $33.97 \pm 1.92$ & $24.50 \pm 1.17$ & $15.80 \pm 0.64$ & $9.67 \pm 0.38$ \\
\hline$X_{u} e \nu$ efficiency $(\%)$ & $43.2 \pm 0.3$ & $42.3 \pm 0.3$ & $41.3 \pm 0.4$ & $40.4 \pm 0.5$ \\
\hline \hline
\end{tabular}

the lepton momenta by the energy ratio to compensate for the difference.

The relative normalization for the two data sets is

$$
r_{L}=\frac{s_{O F F}}{s_{O N}} \frac{\int L_{O N} d t}{\int L_{O F F} d t}=8.433 \pm 0.004 \pm 0.021,
$$

where $s$ and $L$ refer to the c.m. energy squared and luminosity of the two data sets. The statistical uncertainty of $r_{L}$ is determined by the number of detected $\mu^{+} \mu^{-}$pairs used for the $\int L d t$ measurement; the systematic error of the ratio is estimated to be $0.25 \%$.

The $\chi^{2}$ for the fit to the non- $B \bar{B}$ events is defined as follows,

$$
\chi_{c}^{2}=\sum_{i} \frac{\left(f\left(\vec{a}, p_{i}\right)-r_{L} n_{i}\right)^{2}}{r_{L}^{2} n_{i}}+\sum_{j\left(p_{j}>2.8 \mathrm{GeV} / c\right)} \frac{\left(f\left(\vec{a}, p_{j}\right)-N_{j}\right)^{2}}{N_{j}} .
$$

Here $N_{j}$ and $n_{i}$ refer to the number of selected events on- and off-resonance in the $j$-th or $i$-th momentum bin, and $\vec{a}$ is the set of free parameters of the fit. $f(\vec{a}, p)$ is the function approximating the momentum spectrum, for which we have chosen an exponential expression of the form

$$
f(\vec{a}, p)=a_{1}+\exp \left(a_{2}+a_{3} p+a_{4} p^{2}\right),
$$

The fit describes the data well, $\chi^{2}=70$ for 58 degrees of freedom. Above $2.8 \mathrm{GeV} / c$, we observe $(36.7 \pm 0.2) \times 10^{3}$ events in the on-resonance data, whereas the fit predicts $(36.6 \pm 0.2) \times 10^{3}$ events.

\subsection{2 $B \bar{B} \quad$ Background}

The electron spectrum from $B$-meson decays is composed of several contributions, dominated by the various semileptonic $B$-meson decays. Hadronic $B$ decays contribute mostly via hadron misidentification and secondary electrons from decays of $D, J / \psi$, and $\psi^{\prime}$ mesons.

We estimate the total background by fitting the observed inclusive electron spectrum to the sum of the signal and individual background contributions. For the individual signal and $B \bar{B}$ background contributions, we use the Monte Carlo-simulated spectra and treat their relative normalization 
factors as free parameters in the fit. The non- $B \bar{B}$ background is parameterized by the exponential function $f\left(\vec{a}, p_{i}\right)$, as described above. We expand the $\chi^{2}$ definition as follows,

$$
\chi^{2}=\sum_{i} \frac{\left(f\left(\vec{a}, p_{i}\right)-r_{L} n_{i}\right)^{2}}{r_{L}^{2} n_{i}}+\sum_{j} \frac{\left(f\left(\vec{a}, p_{j}\right)+S\left(\vec{b}, p_{j}\right)-N_{j}\right)^{2}}{N_{j}+\sigma_{j M C}^{2}},
$$

where the first sum is for the off-resonance data and the second sum for the on-resonance data. The

$B \bar{B}$ electron spectrum is approximated as $S\left(\vec{b}, p_{j}\right)=\sum_{k} b_{k} g_{k}\left(p_{j}\right)$, where the six free parameters $b_{k}$ are the correction factors to the default branching fractions for the individual contributions $g_{k}\left(p_{j}\right)$ representing the signal $B \rightarrow X_{u} e \nu$ decays, the background $B \rightarrow D e \nu, B \rightarrow D^{*} e \nu, B \rightarrow D^{* *} e \nu$, $B \rightarrow D^{(*)} \pi e \nu$ decays, and the sum of other backgrounds with either electrons from secondary decays or misidentified hadrons. $\sigma_{j M C}$ is a statistical error of the number of simulated events in the $j$-th bin. The momentum spectra $g_{k}\left(p_{j}\right)$ are histograms from Monte Carlo simulations. For the largest contribution, the $B \rightarrow D^{*} e \nu$ decay, we have adjusted the spectrum using the recent BABAR measurements of the form factor parameters [20].

\subsubsection{Fit to Inclusive Spectra}

The fit is performed for the electron-momentum range from $1.1 \mathrm{GeV} / c$ to $3.5 \mathrm{GeV} / c$, in bins of $50 \mathrm{MeV} / c$. The lower part of the spectrum determines the relative normalization of the various background contributions, allowing for an extrapolation into the endpoint region above $2.0 \mathrm{GeV} / c$. To reduce a potential systematic bias from the assumed shape of the signal spectrum, we combine the on-resonance data for the interval from 2.2 to $2.8 \mathrm{GeV} / c$ into a single bin. The lower limit of this bin is chosen so as to retain the sensitivity to the steeply falling $B \bar{B}$ background distributions, while containing a large fraction of the signal events in a region where the background is low. As the limit is lowered to $2.0 \mathrm{GeV} / c$, the resulting error on the background subtraction increases.

The results of the fit and the subtraction of the fitted non- $B \bar{B}$ and $B \bar{B}$ backgrounds are shown in Fig. 1, and compared to Monte Carlo simulations. Above $2.3 \mathrm{GeV} / c$, the non- $B \bar{B}$ background is dominant, while at low momenta the semileptonic $B \bar{B}$ background dominates. Contributions from hadron misidentification are small, varying from $6 \%$ to $4 \%$ as the electron momentum increases. The fit has a $\chi^{2}$ of 100 for 75 degrees of freedom.

Table 1 shows a summary of the data, principal backgrounds and the resulting signal. The errors are statistical, but for the non- $B \bar{B}$ and $X_{c} e \nu$ background they include the uncertainties of the fitted parameters. The data are shown for four different signal regions, ranging in width from $600 \mathrm{MeV} / c$ to $300 \mathrm{MeV} / c$. We choose $2.6 \mathrm{GeV} / c$ as the upper limit of the signal regions because at higher momenta the signal contributions are very small compared to the non- $B \bar{B}$ background. The number of signal events in a given signal interval is taken as the excess of events above the fitted background.

\section{Systematic Errors}

The principal systematic errors originate from the fits to the backgrounds, due to the uncertainties in the simulated momentum spectra of the various contributions. The uncertainty in the event simulation and thereby the impact of the event selection on the momentum dependence of the efficiencies for signal and background are the experimental limitations of the current analysis. In addition, there are smaller corrections to the momentum spectra due to variations in the beam 
energies, and radiative effects. A summary of the systematic errors is given in Table 2 for four intervals in the electron momentum.

Table 2: Summary of the relative systematic errors (\%) on the partial branching fraction measurements for $B \rightarrow X_{u} e \nu$ decays.

\begin{tabular}{lcccc}
\hline \hline$\Delta p(\mathrm{GeV} / c)$ & $2.0 \div 2.6$ & $2.1 \div 2.6$ & $2.2 \div 2.6$ & $2.3 \div 2.6$ \\
\hline Electron identification & 0.8 & 0.8 & 0.8 & 0.8 \\
Track finding efficiency & 0.7 & 0.7 & 0.7 & 0.7 \\
$B \rightarrow X_{u} e \nu$ spectrum & 7.9 & 5.3 & 3.2 & 1.9 \\
Event selection efficiency & 6.4 & 6.7 & 6.3 & 5.7 \\
Non- $B \bar{B}$ background & 2.1 & 2.1 & 2.4 & 2.6 \\
$B \rightarrow D^{*} l \nu$ form factor & 1.7 & 2.6 & 1.7 & 0.8 \\
$B \rightarrow D l \nu$ form factor & 0.3 & 0.9 & 0.8 & 0.7 \\
$B \rightarrow D^{* *} e \nu$ spectrum & 2.3 & 2.0 & 2.6 & 1.3 \\
$J / \psi$ and $\psi^{\prime}$ background & 0.8 & 0.7 & 0.7 & 0.5 \\
Other $e^{ \pm}$background & 0.5 & 0.2 & 0.1 & 0.1 \\
$\pi$ mis-ID background & 0.7 & 0.7 & 0.8 & 0.7 \\
$K$ mis-D background & 0.4 & 0.3 & 0.2 & 0.2 \\
Other hadron mis-ID background & 0.2 & 0.2 & 0.1 & 0.1 \\
$B \rightarrow X_{u} e \nu$ background & 1.2 & 0.7 & 0.3 & 0.1 \\
$B$ momentum & 1.1 & 1.5 & 1.3 & 0.5 \\
$N_{B \bar{B}}$ normalization & 1.1 & 1.1 & 1.1 & 1.1 \\
Bremsstrahlung and FSR correction & 0.8 & 1.1 & 1.2 & 0.7 \\
\hline Total Systematic Error & 11.1 & 9.8 & 8.5 & 7.0 \\
\hline \hline
\end{tabular}

\subsection{Detection and Simulation of $B \rightarrow X_{u} e \nu$ Decays}

The detection efficiency for $B \rightarrow X_{u} \ell \nu$ decays is determined by Monte Carlo simulation. We include in the uncertainty of the signal spectrum not only the uncertainty in simulation of the detector response, but also the uncertainty in the simulation of the momentum and angular distributions of the charged lepton, as well as the hadrons and neutrinos.

\subsubsection{Detector related uncertainties}

For a specific model of the signal decays there are three major factors that determine the efficiency: the track reconstruction for the electron, the electron identification, and losses due to the detector acceptance and the event selection.

The uncertainty in the tracking efficiency has been studied in detail and is estimated to be $\sim 0.7 \%$ per track. The average identification efficiency for electrons above $1.0 \mathrm{GeV} / c$ is estimated to be $91.5 \%$ [13], based on large samples of radiative Bhabha events and two-photon interactions. In $B \bar{B}$ events the actual efficiencies are slightly lower due to higher track multiplicity. This difference decreases gradually from about $2.5 \%$ at $1.0 \mathrm{GeV} / c$ to less than $0.8 \%$ above $2.0 \mathrm{GeV} / c$. An independent estimate of $0.6 \%$ for this uncertainty was derived from a comparison of the efficiency in data and simulation, for electrons from $J / \psi K^{(*)}$ decays. We adopt an error of $2 \%$ for the systematic uncertainty in the electron-identification efficiency at $1.0 \mathrm{GeV} / c$, decreasing to $0.8 \%$ 
above $2.0 \mathrm{GeV} / c$. In addition, we take into account the impact of the momentum-dependent uncertainty of electron identification efficiency on the observed electron spectrum for both signal and background (see below).

\subsubsection{Uncertainties in the signal spectrum}

The momentum distributions of the signal electrons are not precisely known because many of the $B \rightarrow X_{u} \ell \nu$ decay modes are still unobserved or poorly measured due to small event samples, and even for observed ones the decay form factors are not measured. For decays with low mass charmless hadrons the simulation is based on ISGW2 model. For decays to higher mass, mostly non-resonant states we rely on the model of de Fazio and Neubert [16] and a fragmentation model [17].

To evaluate the sensitivity of the signal efficiency to the decay multiplicity and the angular and momentum distributions, we randomly vary the individual branching ratios for decays to resonant and non-resonant charmless hadrons by $50 \%$, except for $B \rightarrow \pi \ell \nu$ and $B \rightarrow \rho \ell \nu$ which are currently measured to $30 \%$ and $25 \%$, respectively. We observe changes of less than $3.0 \%$ for the whole spectrum, and less than $1.0 \%$ in the signal yield above $2.3 \mathrm{GeV} / c$.

The systematic uncertainties inherent in the modeling of the signal decays to non-resonant hadronic states have been studied by varying the shape-function parameters as determined from the measurement of the inclusive photon spectrum [6]. These variations translate to changes of the signal lepton spectrum and thus impact the fit to the overall spectrum. The resulting changes in the signal branching fraction have been evaluated, taking into account the errors and correlation of the shape function parameters.

Not included in this estimate is the sensitivity to the event selection criteria, specifically those based on the variables $R_{2}$ and $p_{\text {miss }}$. These criteria not only influence the signal, but more so the background distributions, and they are discussed below.

\subsection{Non- $B \bar{B}$ Background}

Systematic errors in the subtraction of the non- $B \bar{B}$ background could be introduced by the choice of the fitting function describing the lepton spectrum and by the uncertainty in the relative normalization of the on- and off-resonance data.

To assess the uncertainty in the shape of this background we have compared fits with different parameterizations of the fitting function. In addition to the exponential function described above, we have tried linear combinations of Chebyshev polynomials up to the fifth order. The resulting fits are equally consistent with the data. The differences in the non- $B \bar{B}$ background estimates between different parameterizations are at a level of less than $0.5 \%$.

If relative normalization is treated as a free fit parameter, its deviation from the value based on luminosity measurements is less than one standard deviation, which is equal to $1.5 \%$. Thus for the normalization the more accurate value based on luminosity measurements is used. As a systematic error of non- $B \bar{B}$ background we take $0.5 \%$ of this background contribution which includes the errors of normalization factor and background shape approximation.

\section{$4.3 \quad B \rightarrow J / \psi X$ Background}

$J / \psi$ and $\psi^{\prime}$ decays to lepton pairs are vetoed by a restriction on the di-lepton effective mass. However, this veto is only about $50 \%$ efficient, mostly because of acceptance losses. The remaining, mostly single track background is estimated from simulation. We observe a difference of $5.0 \pm 2.7 \%$ 
between the veto efficiency for lepton pairs in data and simulation, and thus assign a $5 \%$ error to the residual background. Since this background amounts to $18 \%(10 \%)$ of the signal for $p_{e}>2.0 \mathrm{GeV} / c$ $\left(p_{e}>2.3 \mathrm{GeV} / c\right)$ for both differential and integrated spectra, the resulting uncertainty on the signal branching fraction is estimated to vary from $0.8 \%$ to $0.5 \%$.

\section{4 $B \bar{B}$ Background}

A major concern in this analysis is the uncertainty in the estimate of the signal events from $B \rightarrow X_{u} \ell \nu$ decays obtained from the fit of the sum of various background contributions to the observed spectrum. The shapes of $B \bar{B}$ backgrounds are derived from Monte Carlo simulation. The branching fractions for exclusive semileptonic $B \rightarrow X_{c} \ell \nu$ decays are currently not precisely known. Thus the lepton spectra from $B \rightarrow X_{c} \ell \nu$ decays may differ from those of the simulation. For this reason we have introduced scale factors in the fits to the spectrum to adjust the relative normalization of the various contributions. To test the sensitivity to the shape of the dominant contributions, we have varied the form factor for decays to $D^{*} e \nu$ and $D e \nu$, and changed the relative proportion of contributions from narrow and wide resonances to $D^{* *} e \nu$ decays.

The differential decay rate for $B \rightarrow D^{*} \ell \nu$ can be described by three amplitudes, which depend on

the three parameters: $\rho^{2}, R_{1}$, and $R_{2}$. Their measured values are $\rho^{2}=0.769 \pm 0.039 \pm 0.019 \pm 0.032$, $R_{1}=1.328 \pm 0.055 \pm 0.025 \pm 0.025$, and $R_{2}=0.920 \pm 0.044 \pm 0.020 \pm 0.013$ [20]. The quoted errors are statistical uncertainties from the data and Monte Carlo samples, and systematic errors, respectively. The $B \rightarrow D \ell \nu$ differential decay rate can be described by a single parameter $\rho_{D}^{2}$. To study the impact of form factor variations we reweight the Monte Carlo-simulated spectrum for a given decay mode with the relative change of the generator-level spectrum due to changes in the form-factor parameters, and repeat the standard fit to the data. From the observed changes in the signal rate as a function of the choice of the form-factor parameters for $D^{*} e \nu$ decays, we assess the systematic error on the signal rate by taking into account the measured form-factor parameters, their errors, and their covariance matrix [20]. For Dev decays, we rely on a measurement by the CLEO Collaboration [23], $F_{D}(w) / F_{D}(1)=1-\rho_{D}^{2}(w-1)$, where $\rho_{D}^{2}=0.76 \pm 0.16 \pm 0.08$ is the linear slope of the $w$-dependence. The variable $w$ is the product of the four-vector velocities of the $B$ and $D$ mesons and corresponds to the relativistic boost of the $D$ meson in the $B$ rest frame. To estimate the impact of the uncertainty in $\rho_{D}^{2}$ we likewise compare the default fit results with fits performed with a reweighted lepton spectrum. In this case, we replace the default simulation based on the ISGW2 model with the simpler form factor formulation and vary the measured value of $\rho_{D}^{2}$ by one standard deviation. We adopt the mean shift of the signal rate as a systematic error.

To assess the impact of the poorly known branching fraction for various $D^{* *} e \nu$ decay modes on the shape of the lepton spectrum we have repeated the fit with the different relative branching fractions for the individual decays modes. As long as we do not eliminate the decays to the two narrow resonances, $D_{1}(2437)$ and $D_{2}(2459)$, we obtain reasonable results. Specifically, if we eliminate the decays involving the two wider resonances, $D_{0}(2308)$ and $D_{1}^{\prime}(2460)$, the results change by less than $3 \%$. We adopt this change as the estimate of the systematic error due to the uncertainty of decays to $D^{* *}$ states.

Similarly, we vary the branching ratios for secondary electrons from semileptonic $D$ decays by $10 \%$ and adopt the observed change as a systematic error. There is a small background from events which contain a $B \rightarrow X_{u} e \nu$ decay but contribute to the background rather than the signal. We estimate the uncertainty in this contribution to be $30 \%$.

For background from hadronic $B$ decays, the uncertainty in the spectrum is primarily due to the uncertainty in the momentum-dependent hadron misidentification. The uncertainties of misidenti- 
fication probabilities are estimated to be $20 \%, 30 \%$, and $50 \%$ for pions, kaons, and protons respectively. The uncertainty in the fractions of pions, kaons, and proton is taken as a difference between simulated and observed charged particle spectra, which is about $5 \%$ for pions and kaons, and $50 \%$ for much smaller contribution from protons and antiprotons. With these uncertainties in the hadron misidentification backgrounds, the fractional error in the number of subtracted background events is $\sim 20 \%$ for pions, $\sim 30 \%$ for kaons, and $\sim 70 \%$ for protons.

\subsection{Uncertainty in the $B$ Meson Momentum Spectrum}

The non-zero momentum of the $B$ mesons in the $\Upsilon(4 S)$ rest frame affects the shape of the electron spectra near the endpoint. To estimate the systematic error of the inclusive lepton spectra associated with the uncertainty of initial $B$ meson momentum spectrum we compare the simulated and measured energy spectra for fully reconstructed charged $B$ mesons. The widths of the energy distributions agree well, but in some of the data sets we observed a shift in the central value of up to $2.2 \mathrm{MeV}$ relative to the simulation. We correct the simulation for these shifts, and assess the effect of the uncertainty of $0.13 \mathrm{MeV}$ in this shift.

\subsection{Bremsstrahlung and Radiative Corrections}

For comparison with other experiments and with theoretical calculations the signal spectrum resulting from the fit is corrected for bremsstrahlung in the detector and for final-state radiation. Corrections for QED radiation in the decay process are simulated using PHOTOS [24]. This simulation includes multiple-photon emission from the electron, but does not include electroweak corrections for quarks. The accuracy of this simulation has been compared to analytical calculations performed to $\mathcal{O}(\alpha)[24]$. Based on this comparison we assign an uncertainty of $10-15 \%$ to the PHOTOS correction, leading to an uncertainty in the signal yield of about $1 \%$.

The uncertainty in the energy loss due to bremsstrahlung is determined by the uncertainty in the thickness of the detector material, estimated to be $(0.0450 \pm 0.0014) X_{0}$ at normal incidence. This thickness was verified using Bhabha scattering as a function of the polar angle relative to the beam. The impact of this uncertainty on the signal rate was estimated by calculating the impact of an additional $0.0014 X_{0}$ of material; it is of the order of $1 \%$.

\subsection{Sensitivity to the Event Selection}

We have checked the sensitivity of the fits to the lepton spectrum to changes in the event selection, as well as the momentum dependence of the electron selection efficiencies. These variations of the cuts change the signal efficiency and lead to large variations up to $50 \%$ in the size of the non- $B \bar{B}$ background and up to $20 \%$ in the $B \bar{B}$ background. Though some of the observed changes in signal yield may already be covered by the form factor and other variations, we conclude that these tests do reveal significant changes that have to be accounted for. We interpret the observed changes as inadequacies in the simulation of the Monte Carlo-simulated lepton spectra and adopt the observed changes between the default fits and the looser selection criteria as systematic errors.

The largest variation (5\%) is observed for changes in the restriction of the Fox-Wolfram ratio $R_{2}$ from the default value of 0.5 to 0.4 and 0.6. Other sizable variations are observed for changes in the absolute value and direction of the missing momentum vector. $R_{2}$ and the missing momentum are quantities that are derived from the measured momenta of all charged and neutral particles in the event and are therefore sensitive to even small differences in data and the simulation. We 
consider the chosen variations of the cut variables representative for the error estimation, add the observed changes in quadrature, and include them in the overall systematic error.

\section{Results}

\subsection{Determination of Partial $B \rightarrow X_{u} e \nu$ Branching Fraction}

For a given interval $\Delta p$ in the electron momentum, we calculate the inclusive partial branching fraction $B \rightarrow X_{u} e \nu$ according to

$$
\Delta \mathcal{B}=\frac{N_{t o t}(\Delta p)-N_{b g}(\Delta p)}{2 \epsilon(\Delta p) N_{B \bar{B}}}\left(1+\delta_{\text {rad }}(\Delta p)\right)
$$

Here $N_{t o t}$ refers to the total number of electron candidates detected in the on-resonance data and $N_{b g}$ refers to the total background, from non- $B \bar{B}$ and $B \bar{B}$ events, as determined from the fit to the spectrum. $\epsilon(\Delta p)$ is the total efficiency for detecting a signal electron from $B \rightarrow X_{u} e \nu$ decays (including bremsstrahlung in detector material), and $\delta_{\text {rad }}$ accounts for the distortion of the electron spectrum due to final-state radiation. This is a momentum-dependent correction, derived from the Monte Carlo simulation based on PHOTOS [24]. The total number of produced $B \bar{B}$ events is $N_{B \bar{B}}=(88.36 \pm 0.02 \pm 0.97) \times 10^{6}$.

The differential branching fraction as a function of the electron momentum in the $\Upsilon(4 S)$ rest frame is shown in Fig. 2, fully corrected for efficiencies and radiative effects. Partial branching fractions for the four different momentum intervals are summarized in Table 3. There is excellent agreement with the preliminary BABAR measurement [11] and also with a measurement by the CLEO Collaboration [10].

Table 3: Preliminary results on the partial $(\Delta \mathcal{B})$ and the total branching fraction $(\mathcal{B})$ for inclusive $B \rightarrow X_{u} e \nu$ decays for four momentum intervals. $f_{u}(\Delta p)$ is a fraction of the lepton spectrum, that falls into the $\Delta p$ momentum interval. The values of $f_{u}(\Delta p)$ were derived by the CLEO Collaboration [10] from the shape function parameters based on the measurement of the $b \rightarrow s \gamma$ spectrum.

\begin{tabular}{ccccc}
\hline \hline$\Delta p(\mathrm{GeV} / c)$ & $\Delta \mathcal{B}\left(10^{-3}\right)$ & $f_{u}(\Delta p)$ & $\mathcal{B}\left(10^{-3}\right)$ & $\left|V_{u b}\right|\left(10^{-3}\right)$ \\
\hline $2.0 \div 2.6$ & $0.480 \pm 0.029 \pm 0.053$ & $0.278 \pm 0.043 \pm 0.030$ & $1.73 \pm 0.22 \pm 0.33$ & $3.94 \pm 0.25 \pm 0.37 \pm 0.19$ \\
$2.1 \div 2.6$ & $0.355 \pm 0.018 \pm 0.035$ & $0.207 \pm 0.037 \pm 0.027$ & $1.71 \pm 0.19 \pm 0.38$ & $3.93 \pm 0.22 \pm 0.43 \pm 0.19$ \\
$2.2 \div 2.6$ & $0.235 \pm 0.010 \pm 0.020$ & $0.137 \pm 0.025 \pm 0.023$ & $1.72 \pm 0.16 \pm 0.42$ & $3.93 \pm 0.19 \pm 0.48 \pm 0.19$ \\
$2.3 \div 2.6$ & $0.149 \pm 0.006 \pm 0.010$ & $0.078 \pm 0.015 \pm 0.016$ & $1.90 \pm 0.15 \pm 0.52$ & $4.14 \pm 0.16 \pm 0.56 \pm 0.20$ \\
\hline \hline
\end{tabular}

\subsection{Extraction of the Total Charmless Branching Fraction and $\left|V_{u b}\right|$}

To determine the charmless semileptonic branching fraction $\mathcal{B}\left(B \rightarrow X_{u} e \nu\right)$ from the partial branching fraction $\Delta \mathcal{B}(\Delta p)$, one needs to know the fraction $f_{u}(\Delta p)$ of the spectrum that falls into the momentum interval $\Delta p$. The CLEO Collaboration [10] has used the measurement of the inclusive photon spectrum from $b \rightarrow s \gamma$ transitions to derive the parameters describing the shape function to determine the fraction $f_{u}(\Delta p)$, and to estimate the uncertainties of the resulting shape function 


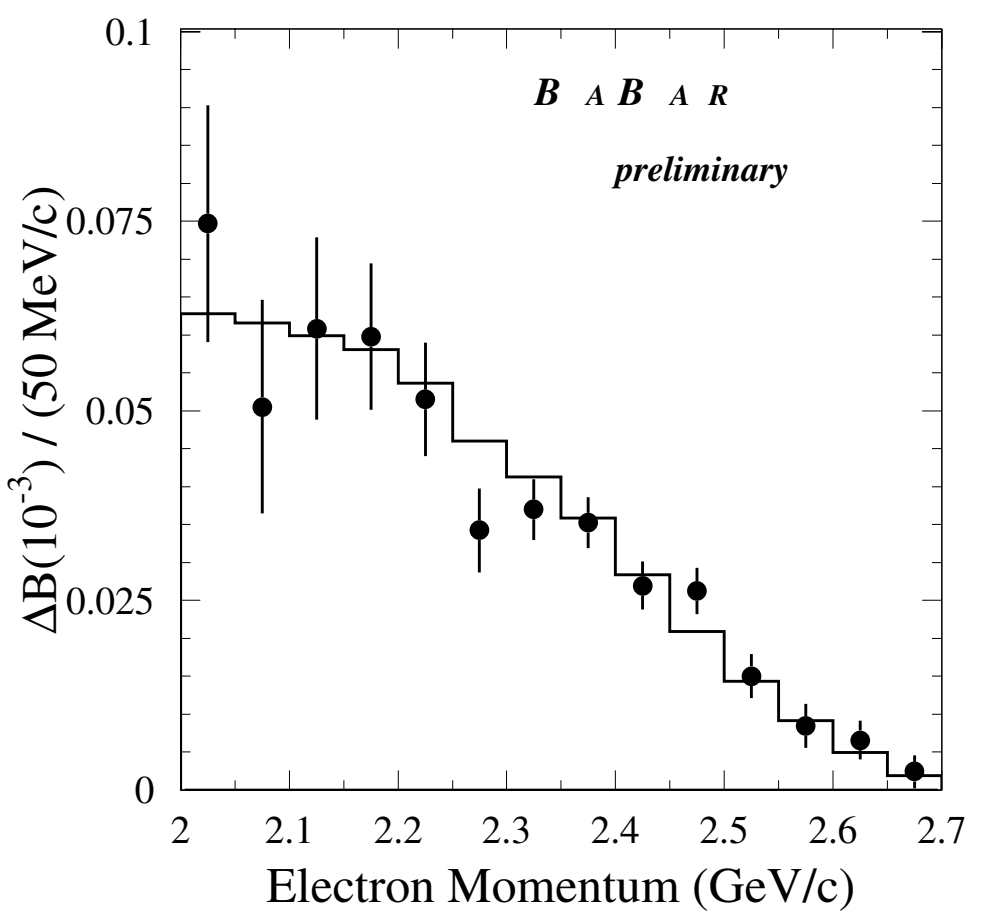

Figure 2: The differential branching fraction for charmless semileptonic $B$ decays (data points) as a function of the electron momentum (in the $\Upsilon(4 S)$ rest frame) after background subtraction and corrections for bremsstrahlung and final state radiation, compared to the Monte Carlo simulation (histogram). The errors indicate the statistical errors on the background subtraction, including the uncertainties of the fit parameters.

parameters. The $f_{u}(\Delta p)$ fractions are listed in Table 3, together with the total branching fractions. Contrary to the CLEO analysis, we have fully corrected the $\Delta \mathcal{B}$ for radiative effects, and consequently the fractions $f_{u}$ listed here do not include such corrections [25]. The quoted errors on $\mathcal{B}$ and $f_{u}$ are statistical and systematic. The systematic error of $f_{u}$ includes the systematic error of $B \bar{B}$ subtraction, uncertainties of the shape function approximation, dependence on $\alpha_{s}$ scale, and the uncertainty of theoretical prediction of $f_{u}$ from $b \rightarrow s \gamma$ shape function. The errors on $\mathcal{B}$ and $\left|V_{u b}\right|$ are the total error from $\Delta \mathcal{B}$ measurement and the error from $f_{u}$. The last error in $\left|V_{u b}\right|$ is the theoretical uncertainty of the translation from $\mathcal{B}$ to $\left|V_{u b}\right|$. Surprisingly, the overall precision does not depend very strongly on the chosen momentum interval. While the experimental errors are smallest for the interval from 2.3 to $2.6 \mathrm{GeV} / c$, the dominant uncertainty arises from the determination of the fraction $f_{u}$ and this increases substantially with higher momentum cut-off. Thus we quote as the branching fraction measurement the result based on the data in the interval $2.0-2.6 \mathrm{GeV} / c$,

$$
\mathcal{B}\left(B \rightarrow X_{u} e \nu\right)=\left(1.73 \pm 0.22_{e x p} \pm 0.33_{f_{u}}\right) \times 10^{-3},
$$

where the first error represents the sum of the statistical and systematic error on the partial branching fraction, and the second error refers to the uncertainty in the measured photon spectrum and the extraction of the shape function. From the inclusive charmless semileptonic branching 
fraction and the average $B$ lifetime we can extract $V_{u b}$,

$$
\left|V_{u b}\right|=0.00424\left(\frac{\mathcal{B}\left(B \rightarrow X_{u} l \nu\right)}{0.002} \frac{1.604 \mathrm{ps}}{\tau_{b}}\right)^{1 / 2}\left(1.0 \pm 0.028_{O P E} \pm 0.039_{m_{b}}\right) .
$$

Here, we rely on a formulation of [26] and [27], taking into account recent measurements of the $B$ lifetime of $1.604 \pm 0.012 \mathrm{ps}$ [28], the $b$ quark mass in the kinetic mass scheme, $m_{b}(1 \mathrm{GeV})=$ $4.61 \pm 0.07 \mathrm{GeV}$, and other parameters of the heavy quark expansions [29]. The first error represents the linear sum of the perturbative and non-perturbative QCD corrections, the second error is due to the uncertainty in $m_{b}$. An overall correction of $0.7 \%$ is included to account for QED corrections. The results for the four momentum intervals are presented in Table 3. They are consistent with each other. For the interval from 2.0 to $2.6 \mathrm{GeV} / c$ we quote as a preliminary result,

$$
\left|V_{u b}\right|=\left(3.94 \pm 0.25_{\exp } \pm 0.37_{f_{u}} \pm 0.19_{\text {theory }}\right) \times 10^{-3} .
$$

Here the first error represents the total experimental uncertainty, and the second refers to the uncertainty on the determination of the fraction $f_{u}$ from the $B \rightarrow X_{s} \gamma$ decays (taken from the CLEO analysis), and the third combines the stated theoretical uncertainties in the extraction of $\left|V_{u b}\right|$ from the branching ratio. No additional uncertainty due to the theoretical assumption of quark-hadron duality has been assigned.

Recently new preliminary determination of the shape function parameters based on analysis of $b \rightarrow s \gamma$ spectrum by the Belle collaboration became available [30] with the central values of $a=2.27$ and $m_{b}^{S F}=4.62 \mathrm{GeV} / c^{2}$. The Belle shape-function parameters differ from those of CLEO by more than one standard deviation. The results for $B \rightarrow X_{u} e \nu$ partial and total branching ratios based on this new set of shape-function parameters and their errors are presented in the Table 4 . The quoted errors on $\Delta \mathcal{B}$ are statistical and systematic, respectively. The total error on $f_{u}$ includes the statistical and systematic errors of the shape-function measurement and the uncertainty of the theoretical calculation of $f_{u}$ from $b \rightarrow s \gamma$ shape function, estimated as in [10]. The errors on $\mathcal{B}$ and $\left|V_{u b}\right|$ are the total error from $\Delta \mathcal{B}$ measurement and the error from $f_{u}$, respectively. The last error in $\left|V_{u b}\right|$ is the theoretical uncertainty of the translation from $\mathcal{B}$ to $\left|V_{u b}\right|$.

Table 4: Preliminary results on the partial $(\Delta \mathcal{B})$ and the total branching fraction $(\mathcal{B})$ for inclusive $B \rightarrow X_{u} e \nu$ decays for four momentum intervals. The recent Belle measurement of $b \rightarrow s \gamma$ spectrum was used [30].

\begin{tabular}{ccccc}
\hline \hline$\Delta p(\mathrm{GeV} / c)$ & $\Delta \mathcal{B}\left(10^{-3}\right)$ & $f_{u}(\Delta p)$ & $\mathcal{B}\left(10^{-3}\right)$ & $\left|V_{u b}\right|\left(10^{-3}\right)$ \\
\hline $2.0 \div 2.6$ & $0.531 \pm 0.032 \pm 0.049$ & $0.246 \pm 0.031$ & $2.16 \pm 0.24 \pm 0.27$ & $4.40 \pm 0.24 \pm 0.28 \pm 0.21$ \\
$2.1 \div 2.6$ & $0.381 \pm 0.020 \pm 0.034$ & $0.174 \pm 0.026$ & $2.19 \pm 0.23 \pm 0.33$ & $4.44 \pm 0.23 \pm 0.33 \pm 0.21$ \\
$2.2 \div 2.6$ & $0.245 \pm 0.011 \pm 0.020$ & $0.110 \pm 0.022$ & $2.23 \pm 0.21 \pm 0.45$ & $4.47 \pm 0.21 \pm 0.45 \pm 0.22$ \\
$2.3 \div 2.6$ & $0.153 \pm 0.006 \pm 0.011$ & $0.058 \pm 0.017$ & $2.64 \pm 0.22 \pm 0.77$ & $4.87 \pm 0.20 \pm 0.71 \pm 0.23$ \\
\hline \hline
\end{tabular}

In conclusion, we have a preliminary measurement of the differential lepton spectrum for charmless semileptonic $B$ decays above $2.0 \mathrm{GeV} / c$, and have extracted the CKM matrix element $\left|V_{u b}\right|$ with improved experimental accuracy. Further improvements to this measurement are expected from new measurements of the inclusive photon spectrum from $B \rightarrow X_{s} \gamma$ decays and further advances in our theoretical understanding of the shape functions, their relation to the parton-level lepton spectrum, and effects of the fragmentation of the $s$ and $u$ quarks. 


\section{ACKNOWLEDGMENTS}

We would like to thank the CLEO and Belle Collaborations for providing detailed information on the extraction of the shape function parameters from the photon spectrum in $b \rightarrow s \gamma$ transition.

We are grateful for the extraordinary contributions of our PEP-II colleagues in achieving the excellent luminosity and machine conditions that have made this work possible. The success of this project also relies critically on the expertise and dedication of the computing organizations that support BABAR. The collaborating institutions wish to thank SLAC for its support and the kind hospitality extended to them. This work is supported by the US Department of Energy and National Science Foundation, the Natural Sciences and Engineering Research Council (Canada), Institute of High Energy Physics (China), the Commissariat à l'Energie Atomique and Institut National de Physique Nucléaire et de Physique des Particules (France), the Bundesministerium für Bildung und Forschung and Deutsche Forschungsgemeinschaft (Germany), the Istituto Nazionale di Fisica Nucleare (Italy), the Foundation for Fundamental Research on Matter (The Netherlands), the Research Council of Norway, the Ministry of Science and Technology of the Russian Federation, and the Particle Physics and Astronomy Research Council (United Kingdom). Individuals have received support from CONACyT (Mexico), the A. P. Sloan Foundation, the Research Corporation, and the Alexander von Humboldt Foundation.

\section{References}

[1] M. Kobayashi and T. Maskawa, Prog. Theor. Phys. 49, 652 (1973).

[2] M. Shifman and M. Voloshin, Sov. J. Nucl. Phys. 41, 120 (1985); J. Chay, H. Georgi, and B. Grinstein, Phys. Lett. B 247, 399 (1990); I. I. Bigi and N. Uraltsev, Phys. Lett. B 280, 271 (1992).

[3] M. Neubert, Phys. Rev. D 49, 3392 (1994).

[4] A. K. Leibovich, I. Low, and I. Z. Rothstein, Phys. Rev. D 61, 053006 (2000).

[5] M. Neubert, Phys. Lett. B 513, 88 (2001); Phys. Lett. B 543, 269 (2002).

[6] CLEO Collaboration, S. Chen et al., Phys. Rev. Lett. 87, 251807 (2001).

[7] Belle Collaboration, P. Koppenburg et al., hep-ex/0403004.

[8] ARGUS Collaboration, H. Albrecht et al., Phys. Lett. B 234, 409 (1990); Phys. Lett. B 255, 297 (1991).

[9] CLEO Collaboration, R. Fulton et al., Phys. Rev. Lett. 64, 16 (1990); J. Bartelt et al., Phys. Rev. Lett. 71, 4111 (1993).

[10] CLEO Collaboration, A. Bornheim et al., Phys. Rev. Lett. 88, 231803 (2002).

[11] BABAR Collaboration, B. Aubert et al., Contribution to ICHEP02, hep-ex/0207081.

[12] BABAR Collaboration, B. Aubert et al., Nucl. Instr. Meth. A 479, 1 (2002).

[13] BABAR Collaboration, B. Aubert et al., Phys. Rev. D 67031101 (2003). 
[14] Particle Data Group, K. Hagiwara et al., Phys. Rev. D 66, 010001 (2002).

[15] N. Isgur, D. Scora, B. Grinstein, and M. B. Wise, Phys. Rev. D 39, 799 (1989); D. Scora, N. Isgur, Phys. Rev. D 52, 2783 (1995).

[16] F. De Fazio, and M. Neubert, JHEP 9906, 017 (1999).

[17] T. Sjöstrand, Comput. Phys. Commun. 82, 74 (1994).

[18] J. L. Goity and W. Roberts, Phys. Rev. D 51, 3459 (1995).

[19] I. I. Bigi, M. Shifman, and N. G. Uraltsev, Annu. Rev. Nucl. Part. Sci. 47, 591 (1997).

[20] The BABAR Collaboration, B. Aubert et al., Measurement of the $B \rightarrow D^{*}$ Form Factors in the Semileptonic Decay $\bar{B} \rightarrow D^{*+} e^{-} \bar{\nu}$, BABAR-CONF-04/31, Contribution to ICHEP04.

[21] CLEO Collaboration, J. E. Duboscq et al., Phys. Rev. Lett. 76, 3898 (1999).

[22] G. C. Fox and S. Wolfram, Phys. Rev. Lett. 41, 1581 (1978).

[23] CLEO Collaboration, J. Bartelt et al., Phys. Rev. Lett. 82, 3746 (1996).

[24] E. Richter-Was, Phys. Lett. B 303, 163 (1993).

[25] L. Gibbons, hep-ex/0402009, and private communication.

[26] N. Uraltsev, Int. J. Mod. Phys. A 14, 4641 (1999), and private communication.

[27] A. H. Hoang, Z. Ligeti, and A. V. Manohar, Phys. Rev. D 59, 074017 (1999).

[28] Particle Data Group, S. Eidelman et al., Phys. Lett. B 592, 1 (2004).

[29] BABAR Collaboration, B. Aubert et al., Phys. Rev. Lett. 93, 011803 (2004).

[30] A. Limosani and T. Nozaki, hep-ex/0407052 (2004). 